\title{
Heart failure hospitalization risk associated with use of two classes of oral antidiabetic medications: an observational, real-world analysis
}

\author{
Santosh Gautam ${ }^{1 *}$, Abiy Agiro ${ }^{1}$, John Barron ${ }^{1}$, Thomas Power ${ }^{2}$, Harry Weisman ${ }^{3}$ and Jeff White ${ }^{3}$
}

\begin{abstract}
Background: Newer oral antidiabetic drug classes are expanding treatment options for type 2 diabetes mellitus (T2DM); however, concerns remain. The objective was to assess relative risk of heart failure hospitalization of sodiumglucose co-transporter-2 (SGLT2) and dipeptidyl peptidase-4 (DPP4) inhibitors in T2DM patients.

Methods: This retrospective observational study used a national commercially insured claims database. Adults (>18 years) with T2DM newly starting SGLT2 or DPP4 medication between April 2013 and December 2014 were included. Depending on their index fill, patients were grouped into either SGLT2 or DPP4 medication class cohorts. The primary outcome was hospitalization for heart failure and the risk was assessed using Cox regression models. Propensity score matching (1:2 ratio) was used to adjust for potential confounders. Analyses were also stratified by the presence of baseline diabetes complication and age (<65 vs $65+)$.

Results: The matched cohort included 4899 SGLT2 and 9798 DPP4 users. The risk of heart failure hospitalization was lower among SGLT2 users in comparison with matched DPP4 users (2.0\% SGLT2 vs 3.1\% DPP4; adjusted hazard ratio [aHR] 0.68; 95\% confidence interval [CI] 0.54-0.86; $p=.001$ ). However, the stratified analyses revealed no risk difference among the majority of the analyzed patients, i.e., those aged $<65$, which comprised $85 \%$ of the matched cohort $(a \mathrm{HR}=0.78 ; 95 \% \mathrm{Cl} 0.57-1.05 ; \mathrm{p}=.09)$, and those without prior complication, which comprised $69 \%$ of matched cohort $(\mathrm{aHR}=0.83 ; 95 \% \mathrm{Cl} 0.54-1.27 ; \mathrm{p}=0.40)$.

Conclusions: In this real-life analysis, the rate of hospitalizations for heart failure was significantly lower for patients initiating an SGLT2 compared with a DPP4 medication, specifically among older patients and those with diabetes complication.
\end{abstract}

Keywords: SGLT2, DPP4, Type 2 diabetes, OAD, Heart failure, Claims analysis

\section{Introduction}

The rising prevalence of type 2 diabetes mellitus (T2DM) is concerning because of significant morbidity and mortality associated with the condition $[1,2]$. The risk of heart failure alone is four- to fivefold higher among patients with diabetes compared with those without the condition [3], and even more so among men and patients

*Correspondence: sgautam@healthcore.com

${ }^{1}$ HealthCore, Inc., 123 Justison St, Suite 200, Wilmington, DE 19801, USA

Full list of author information is available at the end of the article older than 65 years [4]. Additionally, patients with diabetes face a higher risk of a recurrent heart failure event and worse outcomes, compared with people without diabetes [5]. Current Food and Drug Administration guidelines recommend cardiovascular risk evaluation for drugs intended to treat T2DM [6].

Recently, two relatively new classes of oral antidiabetic drugs (OADs)-sodium-glucose co-transporter 2 (SGLT2) inhibitors and dipeptidyl peptidase-4 (DPP4) inhibitors - have received attention regarding their effect on cardiovascular outcomes [7-13]. SGLT2 inhibitors 
are the latest addition to the T2DM treatment armamentarium and are recommended as second-line treatment after metformin, as are the DPP4 inhibitors [14]. The EMPA-REG OUTCOME clinical trial found no cardiovascular risk concerns with empagliflozin, an SGLT2 inhibitor [13]. In fact, the study showed an improvement in cardiovascular prognosis, as evidenced by a lower rate of composite cardiovascular events compared with placebo, and a lower rate of heart failure-associated hospital admissions [13]. Recently the Food and Drug Administration approved empagliflozin to reduce cardiovascular death among patients with T2DM and cardiovascular diseases [15]. Since the study was published, there has been growing interest to establish whether the beneficial impact is a class-level effect, but limited information is available.

Conversely, cardiovascular outcome studies with DPP4 inhibitors have provided conflicting results. The SAVOR-TIMI 53 trial demonstrated the DPP4 inhibitor saxagliptin was associated with a higher rate of heart failure hospitalization than placebo [10]. However, heart failure hospitalization rates for sitagliptin and alogliptin, also DPP4 inhibitors, were comparable to placebo in the TECOS [9] and EXAMINE trials [12], respectively. Two recently published observational studies concluded DPP4 inhibitors had similar risks of heart failure as other antidiabetic medications $[7,11]$. Some studies even have suggested that DPP4 inhibitors may reduce the risk of heart failure [16-18]. Amid those uncertainties, the Food and Drug Administration has warned that two DPP4 inhibitors, saxagliptin and alogliptin, may increase the risk of heart failure, especially in patients who already have cardiovascular or kidney disease [19].

In light of the limited beneficial evidence on SGLT2 inhibitors and potential risk concerns with some of the DPP4 inhibitors, we undertook this study to examine how these two newer drug classes compare with each other in terms of associated risk of hospitalization for heart failure. This is the first study reporting a direct comparison of SGLT2 and DPP4 medication classes on the risk of heart failure hospitalization.

\section{Methods}

\section{Data source}

A retrospective observational study was conducted using medical and pharmacy claims data from October 2012 to October 2016. The data were obtained from the HealthCore Integrated Research Environment ${ }^{\mathrm{SM}}$, which consists of claims and eligibility files from 14 commercial health plans with members geographically dispersed across the United States. This study was exempt from Institutional Review Board review as the researchers only accessed a non-identifiable dataset in full compliance with relevant provisions of the Health Insurance Portability and Accountability Act of 1996.

\section{Patient cohort identification}

The study included adults (aged 18 years or older) who filled a new prescription for SGLT2 or DPP4 medication classes between April 2013 and December 2014. The earliest prescription fill date was defined as the index date. Depending on their index prescription fill, patients were assigned into either SGLT2 or DPP4 medication class cohorts. As our study aim was to conduct a class-level analysis, we did not analyze individual agents. In addition, sample sizes were too small for some agents to conduct a meaningful analysis. The distribution of agents on index date for each medication class is provided in a supplemental table (Additional file 1: Table S1).

We restricted the cohorts to new users only by excluding those who filled a prescription for SGLT2, DPP4, or glucagon-like peptide-1 (GLP1) inhibitors (to avoid confounding due to GLP1 inhibitors, which, like DPP4 inhibitors, are also incretin-based therapy) during the 6 months prior to index date. Undertaking a new user study design approach in an observational study such as ours is important as prevalent users could introduce substantial bias [20]. All patients had at least 6 months of continuous medical and pharmacy insurance coverage prior to the index date for identification of baseline characteristics. Patients were excluded if they had a diagnosis of type 1 diabetes as neither medication class is indicated for type 1 patients.

\section{Primary outcome and follow up}

The primary outcome was hospitalization for heart failure. Inpatient claims were searched for International Classification of Diseases, Ninth or Tenth Revision, diagnosis codes (ICD-9: 428, ICD-10: I50) to identify patients who encountered a heart failure hospitalization. Patients were followed from index date through October 31, 2016 looking for the outcome occurrence and were censored at the end of the study period or the end of health plan enrollment, whichever came first.

\section{Covariates}

We obtained the following baseline characteristics for each patient based on claims information during the 6 months prior to index date: age, gender, comorbidity (defined by Deyo-Charlson comorbidity index score [21]), provider specialty on index fill, diabetes complications [22] (cardiovascular, neuropathy, nephropathy, retinopathy, peripheral vascular, cerebrovascular, and metabolic complications), history of adverse events (heart failure, renal diseases, urinary tract infection, hypoglycemia, ketoacidosis, acute pancreatitis), prior 
OAD exposure (metformin, sulfonylureas, thiazolidinediones, OAD combinations, meglitinide, alpha-glucosidase inhibitors), medication possession ratio [23] of any OAD (as a proxy for persistent exposure to antidiabetic therapy), insulin use, and other concomitant medications (angiotensin converting enzyme inhibitors/angiotensin receptor blockers, diuretics, mineralocorticoids, beta blockers, statins, and other cardiovascular medications). Clinical conditions were identified using ICD-9 or ICD10 codes and medications were identified using General Product Identifier codes.

\section{Statistical analysis}

Differences in baseline characteristics between the cohorts were compared using $t$ test for continuous and $\chi^{2}$ test for categorical variables. Additionally, we used standardized differences to compare those characteristics. Standardized differences were defined as differences between cohort means or proportion relative to the pooled standard deviation. Standardized differences are a useful measure as they are not sensitive to sample size, unlike traditional tests of statistical significance, and a difference of $10 \%$ or more is generally considered meaningful $[24,25]$. Unadjusted difference in the outcome was estimated using the KaplanMeier analysis and Cox regression.

To account for the baseline differences between the cohorts, we considered the propensity score matching technique. A propensity score for SGLT2 (or DPP4) treatment was calculated using a logistic regression model using all the covariates described above. A matched cohort was created by matching a patient in SGLT2 cohort with two patients in DPP4 cohort based on their propensity scores using a greedy matching algorithm [26]. The balance in baseline characteristics between the two matched cohorts was assessed using standardized differences. The balance was also visually assessed using boxplots of propensity scores. Finally, we ran a Cox regression model within the matched cohorts to calculate the adjusted risks of heart failure hospitalization.

\section{Consistency analysis}

Propensity score matching is a widely used technique known for reducing bias; however, it may impair generalizability by leaving out a substantial number of unmatched patients from the analysis. To overcome this issue, we performed consistency analyses using two different analytical approaches-inverse probability of treatment weighting (IPTW) and marginal structural modeling (MSM). For IPTW analysis, we use the method outlined by Austin [27]. First, we calculated inverse probability of treatment weights using a propensity model that included all of the covariates listed above. The balance in baseline characteristics between two cohorts after applying IPTWs was verified. Then, the IPTWs were used in a weighted Cox proportional hazard model to estimate the adjusted hazard ratio (HR) for heart failure hospitalization.

For the MSM analysis, we followed the method outlined by Fewel et al. [28]. MSMs use inverse probability of treatment weights that take into account the probability of being on treatment as well as the probability of being uncensored once the follow-up begins. MSMs are known for producing unbiased estimates by accounting for time varying confounding or informative censoring in addition to addressing baseline differences between study cohorts [29]. The weights were calculated using baseline covariates along with time-varying GLP1 and insulin use. The calculated weights were used in the Cox regression model to estimate the adjusted hazard ratios.

\section{Sensitivity and subgroup analyses}

To reduce potential biases due to differential exposure and loss to follow-up, we performed two separate sensitivity analyses: (a) by restricting the cohort to those who had at least 90 days of exposure to index medication class, and (b) by restricting the cohort to those who had at least 24 months of continuous health plan enrollment after the index date. Additionally, we carried out subgroup analyses by age ( $<65 \mathrm{vs} \geq 65$ ) and history of any diabetes complication (yes vs no).

All treatment effects estimates were based on an intentto-treat principle and a $\mathrm{p}$ value $<.05$ was deemed statistically significant. Statistical analyses were conducted using Stata 13.0 (StataCorp) and SAS Enterprise Guide 7.1 (SAS, Inc).

\section{Results}

\section{Patient characteristics}

We identified 5467 patients in the SGLT2 and 32,060 in the DPP4 cohorts who were eligible for the study. After matching, there were 4899 members in the SGLT2 cohort and 9798 in the DPP4 cohort.

Before matching, there were considerable differences between the study cohorts. Compared with patients in the DPP4 cohort, those in the SGLT2 cohort were younger (mean age 55 years SGLT2 vs 59 years DPP4, $\mathrm{p}<.001$; Table 1). A higher proportion of patients in the SGLT2 cohort were women $(46.0 \%$ SGLT2 vs $42.9 \%$ DPP4, p < .001) and had fewer comorbidities (mean baseline comorbidity score 1.43 SGLT2 vs 1.69 DPP4, $\mathrm{p}<.001$ ). A lower proportion of patients in the SGLT2 cohort had complications of diabetes at baseline, compared with the DPP4 cohort (32.6\% SGLT2 vs 40.0\% DPP4, $\mathrm{p}<.001)$. Additionally, a lower proportion of 
Table 1 Comparison of baseline demographic and clinical characteristics in two study cohorts before and after matching

\begin{tabular}{|c|c|c|c|c|c|c|}
\hline & \multicolumn{3}{|c|}{ Overall unmatched cohorts } & \multicolumn{3}{|c|}{ Matched cohorts } \\
\hline & $\begin{array}{l}\text { DPP4 } \\
n=32,060\end{array}$ & $\begin{array}{l}\text { SGLT2 } \\
n=5467\end{array}$ & $\begin{array}{l}\text { p value* (standardized } \\
\text { difference, \%) }\end{array}$ & $\begin{array}{l}\text { DPP4 } \\
n=9798\end{array}$ & $\begin{array}{l}\text { SGLT2 } \\
n=4899\end{array}$ & $\begin{array}{l}\text { p value* (standardized } \\
\text { difference, } \% \text { ) }\end{array}$ \\
\hline Age at index date (mean, SD) & $59.0(12.5)$ & $54.6(10.0)$ & $<.001(39 \%)$ & $55.1(0.1)$ & $54.9(0.1)$ & $.19(2 \%)$ \\
\hline Age categories, n (\%) & & & $<.001$ & & & .68 \\
\hline $18-44$ & 3911 (12.2) & $871(15.9)$ & $(11 \%)$ & $1591(16.2)$ & $757(15.5)$ & $(2 \%)$ \\
\hline $45-54$ & $7713(24.1)$ & 1689 (30.9) & $(15 \%)$ & $2942(30.0)$ & $1484(30.3)$ & $(1 \%)$ \\
\hline $55-64$ & $10,386(32.4)$ & $2185(40.0)$ & $(16 \%)$ & $3832(39.1)$ & $1937(39.5)$ & $(1 \%)$ \\
\hline$\geq 65$ & $10,050(31.4)$ & $722(13.2)$ & $(45 \%)$ & $1433(14.6)$ & $721(14.7)$ & $(0 \%)$ \\
\hline Gender, n (\%) & & & $<.001$ & & & .73 \\
\hline Female & $13,744(42.9)$ & $2513(46.0)$ & $(6 \%)$ & $4450(45.4)$ & $2210(45.1)$ & $(1 \%)$ \\
\hline \multicolumn{7}{|l|}{ Comorbidity } \\
\hline $\mathrm{DCl}$, mean (SD) & $1.69(1.5)$ & $1.43(1.4)$ & $<.001(20 \%)$ & $1.4(0.01)$ & $1.4(0.02)$ & $.54(1 \%)$ \\
\hline DCl categories, n (\%) & & & $<.001$ & & & .24 \\
\hline 0 & $2483(7.7)$ & $330(6.0)$ & $(7 \%)$ & $721(7.4)$ & $322(6.6)$ & $(3 \%)$ \\
\hline 1 & $18,075(56.4)$ & $3509(64.2)$ & $(16 \%)$ & $6276(64.1)$ & $3162(64.5)$ & $(1 \%)$ \\
\hline 2 & $5613(17.5)$ & $1014(18.6)$ & $(3 \%)$ & $1729(17.7)$ & $849(17.3)$ & $(1 \%)$ \\
\hline 3 & $5889(18.4)$ & $614(11.2)$ & $(20 \%)$ & $1072(10.9)$ & $566(1.6)$ & $(2 \%)$ \\
\hline $\begin{array}{l}\text { Provider specialty on index } \\
\text { fill, } n(\%)\end{array}$ & & & $<.001$ & & & .96 \\
\hline Endocrinologist & $3327(10.4)$ & $1114(20.4)$ & $(28 \%)$ & $1425(14.5)$ & $710(14.5)$ & $(0 \%)$ \\
\hline PCP & $23,215(72.4)$ & $3382(61.9)$ & $(23 \%)$ & $6581(67.2)$ & $3283(67.0)$ & $(0 \%)$ \\
\hline Other & $5518(17.2)$ & $971(17.8)$ & $(1 \%)$ & $1792(18.3)$ & $906(18.5)$ & $(1 \%)$ \\
\hline \multicolumn{7}{|l|}{ Diabetes complications, n (\%) } \\
\hline Any & $12,816(40.0)$ & $1784(32.6)$ & $<.001(15 \%)$ & $3032(31.0)$ & $1561(31.9)$ & $.26(2 \%)$ \\
\hline Cardiovascular & $6635(20.7)$ & $726(13.3)$ & $<.001(20 \%)$ & $1318(13.5)$ & $671(13.7)$ & $.68(1 \%)$ \\
\hline Neuropathy & $4170(13.0)$ & $751(13.7)$ & $.14(2 \%)$ & $1148(11.7)$ & $616(12.6)$ & $.13(3 \%)$ \\
\hline Nephropathy & $3663(11.4)$ & $312(5.7)$ & $<.001(21 \%)$ & $544(5.6)$ & $290(5.9)$ & $.36(2 \%)$ \\
\hline Retinopathy & $2165(6.8)$ & $336(6.2)$ & $.10(3 \%)$ & $548(5.6)$ & $274(5.6)$ & $1.00(0 \%)$ \\
\hline Peripheral vascular & $1886(5.9)$ & $191(3.5)$ & $<.001(11 \%)$ & $345(3.5)$ & $180(3.7)$ & $.64(1 \%)$ \\
\hline Cerebrovascular & $1580(4.9)$ & $129(2.4)$ & $<.001(14 \%)$ & $273(2.8)$ & $125(2.6)$ & $.41(2 \%)$ \\
\hline Metabolic & $204(0.6)$ & $35(0.6)$ & $0.97(0 \%)$ & $49(0.5)$ & $28(0.6)$ & $.57(1 \%)$ \\
\hline \multicolumn{7}{|l|}{ History of adverse events, n (\%) } \\
\hline Renal & $2618(8.2)$ & $155(2.8)$ & $<.001(24 \%)$ & $297(3.0)$ & $155(3.2)$ & $.66(1 \%)$ \\
\hline UTI & $2106(6.6)$ & $279(5.1)$ & $<.001(6 \%)$ & $509(5.2)$ & $258(5.3)$ & $.85(0 \%)$ \\
\hline $\mathrm{CHF}$ & $1641(5.1)$ & $115(2.1)$ & $<.001(16 \%)$ & $225(2.3)$ & $112(2.3)$ & $.97(0 \%)$ \\
\hline Hypoglycemia & $886(2.8)$ & $157(2.9)$ & $0.65(1 \%)$ & $248(2.5)$ & $130(2.7)$ & $.66(1 \%)$ \\
\hline Ketoacidosis & $101(0.3)$ & $13(0.2)$ & $0.34(2 \%)$ & $22(0.2)$ & $12(0.2)$ & $.81(0 \%)$ \\
\hline Pancreatitis & $98(0.3)$ & $30(0.6)$ & $.004(4 \%)$ & $36(0.4)$ & $14(0.3)$ & $.42(1 \%)$ \\
\hline \multicolumn{7}{|l|}{ Prior OAD use, n (\%) } \\
\hline Any & $24,394(76.1)$ & $4183(76.5)$ & $.50(1 \%)$ & $7496(76.5)$ & $3721(76.0)$ & $.46(1 \%)$ \\
\hline Metformin & $19,700(61.5)$ & $3431(62.8)$ & $.07(3 \%)$ & $6105(62.3)$ & $3084(63.0)$ & $.45(1 \%)$ \\
\hline Sulfonylurea & $10,576(33.0)$ & $1720(31.5)$ & $.03(3 \%)$ & $3056(31.2)$ & $1575(32.2)$ & $.24(2 \%)$ \\
\hline TZD & $1880(5.9)$ & $404(7.4)$ & $<.001(6 \%)$ & $649(6.6)$ & $338(6.9)$ & $.53(1 \%)$ \\
\hline Combination OAD & $1001(3.1)$ & $272(5.0)$ & $<.001(9 \%)$ & $421(4.3)$ & $213(4.4)$ & $.89(0 \%)$ \\
\hline Meglitinide & $370(1.2)$ & $58(1.1)$ & $.55(1 \%)$ & $91(0.9)$ & $52(1.1)$ & $.44(1 \%)$ \\
\hline Alpha-glucosidase inhibitor & $109(0.3)$ & $19(0.4)$ & $.93(0 \%)$ & $25(0.3)$ & $16(0.3)$ & $.44(1 \%)$ \\
\hline MPR of any OAD, mean (SD) & $0.44(0.4)$ & $0.47(0.5)$ & $<.001(10 \%)$ & $0.45(0.004)$ & $0.46(0.005)$ & $.14(3 \%)$ \\
\hline Insulin, n (\%) & $3237(10.1)$ & $1481(27.1)$ & $<.001(45 \%)$ & $1848(18.9)$ & $920(18.8)$ & $.91(0 \%)$ \\
\hline \multicolumn{7}{|l|}{ Other medications, n (\%) } \\
\hline Cardiovascular drugs & $4096(12.8)$ & $445(8.1)$ & $<.001(17 \%)$ & $869(8.9)$ & $403(8.2)$ & $.19(2 \%)$ \\
\hline
\end{tabular}


Table 1 continued

\begin{tabular}{|c|c|c|c|c|c|c|}
\hline & \multicolumn{3}{|c|}{ Overall unmatched cohorts } & \multicolumn{3}{|c|}{ Matched cohorts } \\
\hline & $\begin{array}{l}\text { DPP4 } \\
n=32,060\end{array}$ & $\begin{array}{l}\text { SGLT2 } \\
\mathrm{n}=5467\end{array}$ & $\begin{array}{l}\text { p value* (standardized } \\
\text { difference, } \%)\end{array}$ & $\begin{array}{l}\text { DPP4 } \\
\mathrm{n}=9798\end{array}$ & $\begin{array}{l}\text { SGLT2 } \\
\mathrm{n}=4899\end{array}$ & $\begin{array}{l}\text { p value* (standardized } \\
\text { difference, \%) }\end{array}$ \\
\hline Statin & $18,578(58.0)$ & $3017(55.2)$ & $<.001(6 \%)$ & $5307(54.2)$ & $2696(55.0)$ & $.32(2 \%)$ \\
\hline ACE/ARB & $14,856(46.3)$ & $2418(44.2)$ & $.004(4 \%)$ & $4211(43.0)$ & $2139(43.7)$ & $.43(1 \%)$ \\
\hline Diuretic & $10,991(34.3)$ & $1774(32.5)$ & $.008(4 \%)$ & $3063(31.3)$ & $1585(32.4)$ & $.18(2 \%)$ \\
\hline Spironolactone & $709(2.2)$ & $114(2.1)$ & $0.56(1 \%)$ & $172(1.8)$ & $104(2.1)$ & $.12(2 \%)$ \\
\hline Beta blocker & $9171(28.6)$ & $1292(23.6)$ & $<.001(11 \%)$ & $2250(23.0)$ & $1159(23.7)$ & $.35(2 \%)$ \\
\hline
\end{tabular}

$A C E / A R B$ angiotensin converting enzyme inhibitor/angiotensin receptor blocker, CHF coronary heart failure, DCI Deyo-Charlson comorbidity index, DPP4 dipeptidyl peptidase-4, MPR medication possession ratio, OAD oral antidiabetic drug, PCP primary care provider, SD standard deviation, SGLT2 sodium-glucose co-transporter 2 , TZD thiazolidinediones, UTI urinary tract infection

* $p$ values were derived from $x^{2}$ tests for categorical variables and $t$ tests for continuous variables. Comparison group was DPP4

patients in the SGLT2 cohort had a history of heart failure $(2.1 \%$ SGLT2 vs $5.1 \%$ DPP4, $\mathrm{p}<.001)$.

However, after matching, the characteristics were well balanced as none of them remained significantly different between the two matched cohorts (Table 1). The balance was also confirmed through visual assessment (Additional file 2: Figure S1).

\section{Follow up and outcome}

In the overall unmatched cohort, the median length of follow up for the SGLT2 cohort was 23.0 months (interquartile range 10.6-28.3 months, maximum 42.7 months; Table 2), whereas it was 23.8 months for the DPP4 cohort (interquartile range 9.7-32.0 months, maximum 42.9 months). The follow-up period in the matchedcohorts was comparable to that of overall unmatched cohorts. In terms of exposure, the average number of fills and average days covered were comparable in both the unmatched and matched cohorts.
Table 2 lists and Fig. 1 shows the difference in outcomes between the unmatched and matched cohorts. In the overall unmatched cohorts, patients in the SGLT2 cohort had a lower rate of heart failure hospitalization than patients in the DPP4 cohort (109 patients, $2.0 \%$ SGLT2 vs 1734 patients, $5.4 \%$ DPP4) and the unadjusted HR for the SGLT2 cohort was 0.39 (95\% CI 0.32-0.48; $\mathrm{p}$ <.001) compared with the DPP4 cohort (Table 2).

In the matched cohort, the difference in the rate of heart failure hospitalization decreased slightly (96 patients, 2.0\% SGLT2 vs 307 patients, 3.1\% DPP4). The associated adjusted risk indicated that patients in the SGLT2 cohort were 32\% less likely to have an admission for heart failure than those in the DPP4 cohort (adjusted HR [aHR] 0.68; 95\% CI 0.54-0.86; $\mathrm{p}=.001$ ). Consistency analyses on all patients using IPTW adjusted method yielded an aHR of 0.66 (95\% CI $0.50-0.88 ; \mathrm{p}=.001$; Fig. 2) whereas the MSM model yielded an aHR of 0.60

Table 2 Comparison of unadjusted risk of heart failure hospitalization in two study cohorts before and after matching

\begin{tabular}{|c|c|c|c|c|}
\hline & \multicolumn{2}{|c|}{ Overall unmatched cohorts } & \multicolumn{2}{|l|}{ Matched cohorts } \\
\hline & $\begin{array}{l}\text { DPP4 } \\
\mathrm{n}=32,060\end{array}$ & $\begin{array}{l}\text { SGLT2 } \\
n=5467\end{array}$ & $\begin{array}{l}\text { DPP4 } \\
n=9798\end{array}$ & $\begin{array}{l}\text { SGLT2 } \\
n=4899\end{array}$ \\
\hline \multicolumn{5}{|l|}{ Follow up length (months) } \\
\hline Median & 23.8 & 23.0 & 24.0 & 22.9 \\
\hline 25th, 75th percentiles & $9.7,32.0$ & $10.6,28.3$ & $10.5,32.1$ & $10.5,30.8$ \\
\hline Maximum & 42.9 & 42.7 & 42.9 & 42.7 \\
\hline \multicolumn{5}{|l|}{ Exposure to index medication class } \\
\hline Average number of fills & 10.2 & 11.3 & 10.4 & 11.2 \\
\hline Average number of days covered & 359 & 357 & 365 & 354 \\
\hline Patients w/heart failure hospitalization & 1734 & 109 & 307 & 96 \\
\hline Proportion of patients w/heart failure hospitalization (\%) & 5.4 & 2.0 & 3.1 & 2.0 \\
\hline Person-years of follow up, $\mathrm{n}$ & 58,018 & 9192 & 17,981 & 8228 \\
\hline Event rate $(95 \% \mathrm{Cl})$ per 100 person years & $2.99(2.85-3.13)$ & $1.19(0.98-1.43)$ & $1.71(1.53-1.91)$ & $1.17(0.96-1.43)$ \\
\hline HR (95\% Cl), unadjusted & $0.39(0.32-0.48) ; p<.001$ & & $0.68(0.54-0.86) ; p=.001$ & \\
\hline
\end{tabular}

Cl confidence interval, DPP4 dipeptidyl peptidase-4, HR hazard ratio, SGLT2 sodium-glucose co-transporter 2 


\section{Overall Cohort}

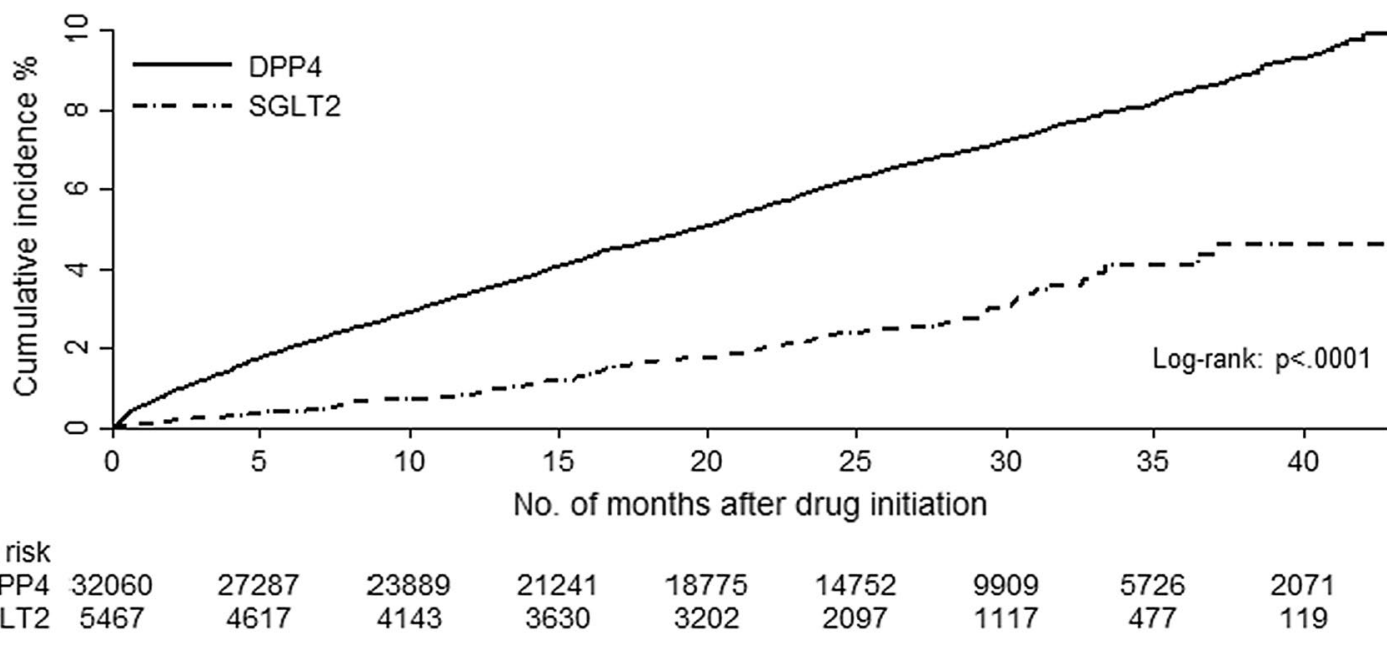

\section{Matched Cohort}

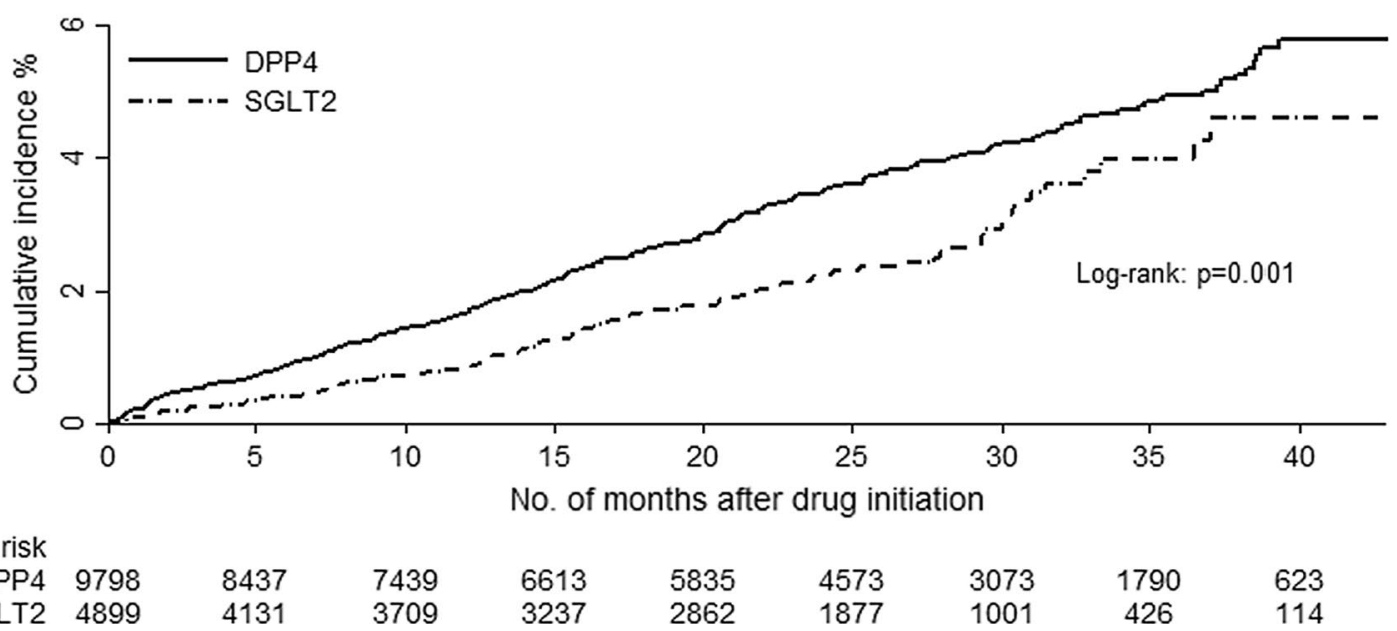

Fig. 1 Cumulative incidence of heart failure hospitalization in two study cohorts before and after matching

(95\% CI 0.50-0.71; $\mathrm{p}<.001)$ for the SGLT2 cohort compared with the DPP4 cohort.

\section{Subgroup analyses}

In the matched cohorts of patients aged 65 years or older, the proportion of patients with heart failure hospitalization was lower in the SGLT2 cohort compared with the matched patients in DPP4 cohort (4.7\% SGLT2 vs 9.1\% DPP4; aHR 0.60; 95\% CI 0.41-0.87; $\mathrm{p}=.008$; Additional file 3: Table S2). Among those younger than 65 years, the risk of heart failure was slightly lower in the SGLT2 cohort compared with the DPP4 cohort; however, the association was not significant (aHR 0.77; 95\% CI
0.57-1.05; $\mathrm{p}<.09$; Additional file 3: Table S2, Additional file 4: Figure S2).

Similarly, the proportion of admissions for heart failure was lower in the SGLT2 cohort compared with the DPP4 cohort among matched cohorts of patients with diabetes complication (4.5\% SGLT2 vs 6.9\% DPP4; aHR 0.68; 95\% CI 0.52-0.90; $\mathrm{p}=.006$; Additional file 3: Table S2). Among the matched cohorts without diabetes complication, a lower proportion of members in the SGLT2 cohort experienced the event; however, the risk was not statistically significant (aHR 0.83; 95\% CI 0.54-1.27; p = 0.40; Additional file 3: Table S2, Additional file 5: Figure S3). We separately assessed and confirmed that age 65 years 


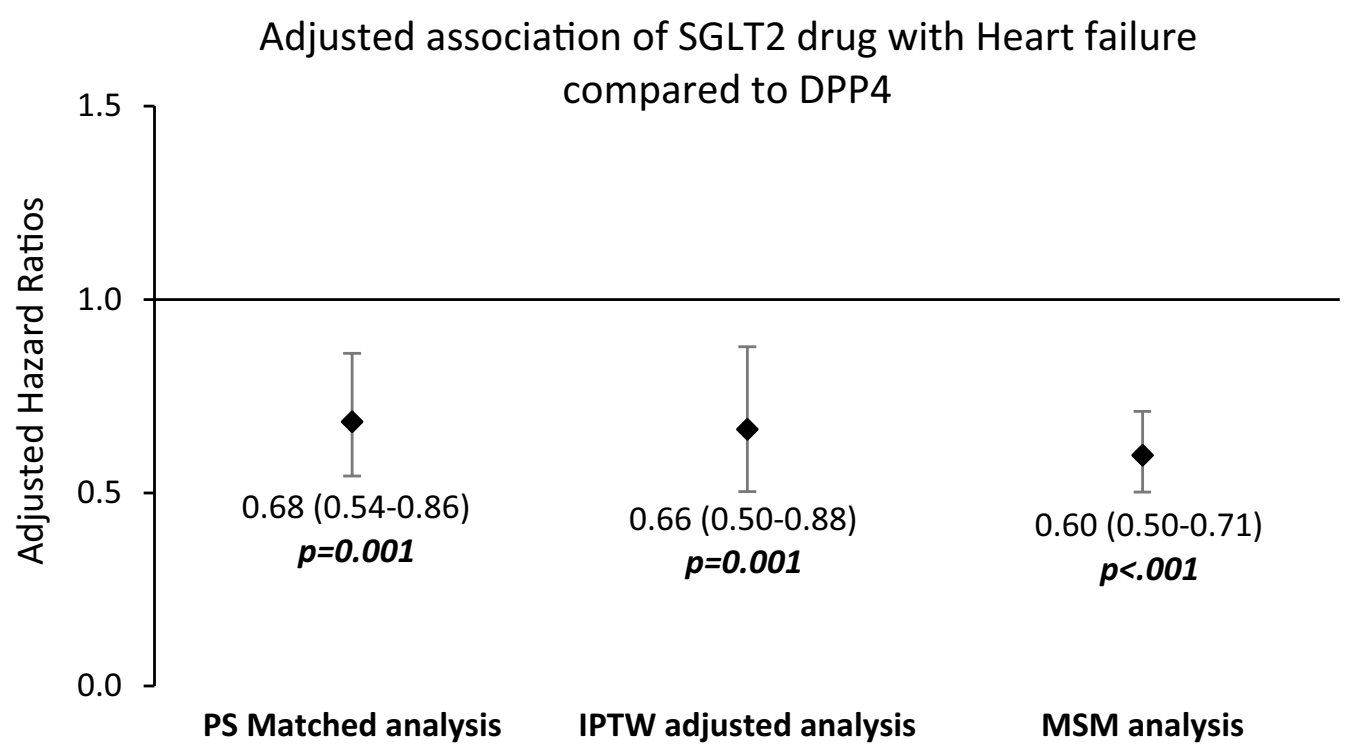

Fig. 2 Adjusted hazard ratios for heart failure hospitalization using different analytical methods in two study cohorts

or older and established diabetes complication are independently associated with heart failure hospitalization.

\section{Sensitivity analyses}

After restricting the matched cohorts to those who had at least 90 days of exposure to the index class drug, the heart failure hospitalization rate still remained lower for the SGLT2 cohort compared with the DPP4 cohort (61 patients, $1.7 \%$ in SGLT2 vs. 182 patients, $2.5 \%$ in DPP4) with aHR 0.74 (95\% CI 0.55-0.98, p = 0.038; Additional file 6: Table S3). We observed a similar trend in the matched cohorts with at least 24 months of post-index enrollment in the health plan. There were 60 patients (2.7\%) in the SGLT2 cohort and 180 patients $(4.1 \%)$ in the DPP4 cohort who experienced a heart failure hospitalization event $(\mathrm{aHR}=0.72,95 \%$ CI 0.54-0.97; $\mathrm{p}=0.030)$.

\section{Discussion}

The goal of this study was to compare the risk of heart failure hospitalization between two relatively new classes of oral antidiabetic agents, DPP4 and SGLT2 inhibitors, among patients with T2DM in a real-world setting. In the propensity score-matched cohorts, we observed a lower adjusted risk of heart failure hospitalization among patients taking SGLT2 inhibitors than those taking DPP4 inhibitors. This result changed slightly and remained significant in our consistency analyses in all patients (including unmatched patients) using different statistical approaches as well as in the sensitivity analyses around medication exposure and follow-up durations. However, our subgroup analyses revealed that there was no difference in heart failure hospitalization risks between the two medication classes among the majority of the analyzed patients, i.e., those younger than 65 years ( $85.3 \%$ of matched cohort) and also in patients without a prior history of diabetes complications $(68.7 \%$ of matched cohort).

\section{DPP4 inhibitors and heart failure}

Heart failure risks associated with several DPP4 inhibitors have been assessed in randomized clinical trials, meta-analyses, and observational studies, producing conflicting results ranging from reduced risk $[3,17,18]$, to no risk $[7,9,11,12,30-32]$, to high risk $[8,16,30,33-$ 35] of heart failure. Such discrepant findings may have stemmed primarily from different comparator groups across these studies as well as differences in study design and population selection. For this very reason, it was difficult to make a head-to-head comparison of our results with those previous studies. However, it is noteworthy that no prior studies directly compared DPP4 inhibitors with SGLT2 inhibitors, and our finding that at a class level DPP4 inhibitors are associated with higher risk of heart failure hospitalization than SGLT2 inhibitors will be an important addition to the literature.

\section{SGLT2 inhibitors and heart failure}

On the other hand, there are few published studies examining the heart failure risk associated with SGLT2 medications, and they have reported more consistent results. For example, the EMPA-REG study, the first randomized clinical trial to examine an SGLT2 inhibitor, found that 
empagliflozin was associated with lower risks of heart failure hospitalization compared with placebo (HR 0.65; 95\% CI 0.50-0.85) [13]. Another recently published clinical trial reported a lower risk of heart failure hospitalization associated with canagliflozin, another SGLT2 inhibitor, compared with placebo (HR 0.67; 95\% CI 0.52, 0.87) [36]. Similar findings were reported in a multinational observational study comparing class level effect of SGLT2 inhibitors to other glucose-lowering drugs with a reported HR of 0.61 (95\% CI 0.51-0.73) [37]. Those findings are consistent with our results; however, such comparisons warrant some caution as our comparator group was DPP4 rather than placebo or any glucose-lowering drugs. Additionally, there were differences in patient risk profile and follow-up time. Nevertheless, our finding is intriguing in light of the overwhelming interest around the reduction of heart failure risks associated with SGLT2 inhibitors.

\section{Potential class level effect and underlying mechanism}

Since publication of the EMPA-REG trial, two important questions have been gaining ground: first, whether the protective effect of SGLT2 on heart failure is a class-level effect [38, 39]; and second, speculation as to the possible underlying mechanism. Recently published studies by Neal et al. and Kosiborod et al. point towards a potential class-level effect of SGLT2 inhibitors [36, 37]. Our study also shows lower rate of heart failure hospitalization associated with each drugs in the SGLT2 class and hence serves as an additional piece of evidence to support the earlier findings (see Additional file 1: Table S1). The results from ongoing trial examining dapagliflozin (Dapagliflozin Effect on CardiovascuLAR Events [40]) remain crucial for establishing the class-level effect of SGLT2 inhibitors on risk of heart failure hospitalization, although a recent meta-analysis suggests that the effect of dapagliflozin on heart failure hospitalization is comparable to empagliflozin [41].

Regarding the question of underlying mechanism, studies that have examined the cardiovascular effect of DPP4 inhibitors are not able to establish a direct link with cardiac or endothelial effect $[16,42]$. On the other hand, studies examining beneficial cardiovascular effect of SGLT2 inhibitors have pointed out several plausible pathways such as glycemic control, body weight reduction, lowering in blood pressure, and reduction in albuminuria, but it is still not clearly understood [36, 39, 43, 44]. Studies mostly agree that the observed cardiovascular effect is unlikely to be coming from a direct effect of SGLT2 on cardiac function [43-46]. Exploring an in-depth understanding of the underlying mechanism is necessary to establish a more robust evidence base. Ongoing clinical trials evaluating cardiovascular effects of SGLT2 inhibitors may shed additional light on it in the future [47].

To the best of our knowledge, this is the first observational study comparing heart failure risks associated with the SGLT2 and DPP4 medication classes. The findings of this study have important implications. These results could prove to be useful to clinicians providing care to patients with T2DM at risk for heart failure and to the patients themselves, as well as to regulatory agencies and professional societies [48, 49]. The strengths of our study include a large sample size, approximately 2 years of average follow up, findings based on equally balanced treatment cohorts, and assessment of the robustness of the results using multiple statistical methods followed by several subgroup and sensitivity analyses.

\section{Limitations}

Several limitations should be considered when interpreting the results of this study. First, this is a class-level analysis of risk differences and should not be extrapolated to agent-level differences. Selection bias may have been present as this study was a comparison of non-randomized treatment groups. We attempted to minimize such biases by balancing our cohorts through propensity score matching using an array of potential confounders. Results were also further verified through consistency analyses that included all patients using IPTW and MSM methods. Yet, residual bias may have remained due to unmeasured confounding from factors such as body mass index, smoking status, and other behavioral health aspects. This information is important in assessing diabetes outcomes, but is rarely available in claims data. Claims data also lack information on diabetes onset or duration, which could potentially affect the outcomes discussed. Additionally, while it is possible to determine that prescriptions for OADs were filled, it is uncertain whether patients took the medication as dispensed. Another potential limitation could be the differential follow-up time in the two cohorts, which could have affected the exposure time and outcomes. To address this limitation, we conducted a sensitivity analysis using a marginal structural model that allowed accounting for censoring and adjusting for time varying confounding [50] and found the results to be similar in terms of trend directionality and statistical significance.

\section{Conclusions}

In this real-world analysis of T2DM patients, our findings suggest that SGLT2 inhibitors are associated with lower risk of heart failure hospitalization than DPP4 inhibitors, specifically among patients older than 65 years of age or those with a prior history of diabetic 
complications. Future studies could focus on comparisons of the SGLT2 class with other antidiabetic drug classes to further confirm the potential class level effect of SGLT2 drugs. Additionally, future studies could also compare individual agents and examine other cardiovascular outcomes, such as myocardial infarction and stroke, to establish more robust evidence around those therapies.

\section{Additional files}

Additional file 1: Table S1. Breakdown of individual agents in each drug class.

Additional file 2: Figure S1. Visual assessment of propensity scoresbefore and after matching.

Additional file 3: Table S2. Heart failure hospitalization—subgroup analysis by age and history of cardiovascular disease.

Additional file 4: Figure S2. Cumulative incidence of heart failure hospitalization among patients younger than 65 years (before and after matching).

Additional file 5: Figure S3. Cumulative incidence of heart failure hospitalization among patients without diabetes complication (before and after matching).

Additional file 6: Table S3. Heart failure hospitalization-sensitivity analysis by medication exposure and outcome follow up.

\begin{abstract}
Abbreviations
aHR: adjusted hazard ratio; Cl: confidence interval; DPP4: dipeptidyl peptidase-4; EMPA-REG OUTCOME: empagliflozin removal of excess glucose outcome trial; EXAMINE: examination of cardiovascular outcomes with alogliptin versus standard of care; GLP-1: glucagon-like peptide-1; HR: hazard ratio; ICD-9, ICD-10: International Classification of Diseases, Ninth Revision; International Classification of Diseases, Tenth Revision; IPTW: inverse probability of treatment weighting; MSM: marginal structural modeling; OAD: oral antidiabetic drug; SAVOR-TIMI 53: saxagliptin assessment of vascular outcomes recorded in patients with diabetes mellitus-thrombolysis in myocardial infarction 53; SGLT2: sodium-glucose co-transporter 2; T2DM: type 2 diabetes mellitus; TECOS: trial evaluating cardiovascular outcomes with sitagliptin.
\end{abstract}

\section{Authors' contributions}

SG conceptualized the study; collected, analyzed, and interpreted the data; and wrote and revised the manuscript. AA conceptualized the study; collected, analyzed, and interpreted the data; and reviewed and revised the manuscript. JB conceptualized the study; analyzed, and interpreted the data; and reviewed and revised the manuscript. TP, HW interpreted the data; and reviewed and revised the manuscript. JW conceptualized the study, analyzed and interpreted the data; and reviewed and revised the manuscript. All authors read and approved the final manuscript.

\section{Author details}

${ }^{1}$ HealthCore, Inc., 123 Justison St, Suite 200, Wilmington, DE 19801, USA. ${ }^{2}$ AIM Specialty Health, Chicago, IL, USA. ${ }^{3}$ Anthem, Inc., Indianapolis, IN, USA.

\section{Acknowledgements}

The authors gratefully acknowledge Dianna Hayden, Christian Rojas-Lazic, and Ramya Avula, HealthCore, Inc., for programming assistance; and Cheryl Jones; HealthCore, Inc., for editorial assistance.

\section{Competing interests}

SG, AA, and JB are employees of HealthCore, Inc., a wholly owned subsidiary of Anthem, Inc. TP is an employee of AIM Specialty Health, a wholly owned subsidiary of Anthem, Inc. HW and JW are employees of Anthem, Inc.

\section{Availability of data}

All data generated relevant to this study were presented in the text and additional material.

\section{Consent for publication \\ Not applicable.}

\section{Ethics approval and consent to participate}

This study was exempt from Institutional Review Board review as the researchers only accessed a non-identifiable dataset in full compliance with relevant provisions of the Health Insurance Portability and Accountability Act of 1996.

\section{Funding}

Funding for this study was provided by Anthem, Inc. Anthem, Inc., had no role in study design; collection, analysis, or interpretation of the data; manuscript preparation or review; or the decision to publish.

\section{Publisher's Note}

Springer Nature remains neutral with regard to jurisdictional claims in published maps and institutional affiliations.

Received: 8 June 2017 Accepted: 21 July 2017

Published online: 31 July 2017

\section{References}

1. National diabetes statistics report, 2014. http://www.cdc.gov/diabetes/ pdfs/data/2014-report-estimates-of-diabetes-and-its-burden-inthe-united-states.pdf. Accessed 8 Nov 2016.

2. Geiss LS, Li Y, Kirtland K, Barker L, Burrows NR, Gregg EW. Increasing prevalence of diagnosed diabetes_-United States and Puerto Rico, 1995-2010. MMWR. 2012;61(45):918-21.

3. Kannel WB, Hjortland M, Castelli WP. Role of diabetes in congestive heart failure: the Framingham study. Am J Cardiol. 1974;34(1):29-34.

4. Ho KK, Pinsky JL, Kannel WB, Levy D. The epidemiology of heart failure: the Framingham study. J Am Coll Cardiol. 1993;22(4 Suppl A):6A-13A.

5. Desai AS, Stevenson LW. Rehospitalization for heart failure: predict or prevent? Circulation. 2012;126(4):501-6.

6. Guidance for industry: diabetes mellitus_evaluating cardiovascular risk in new antidiabetic therapies to treat type 2 diabetes. http://www.fda. gov/ucm/groups/fdagov-public/@fdagov-drugs-gen/documents/document/ucm071627.pdf. Accessed 13 Dec 2016.

7. Filion KB, Azoulay L, Platt RW, Dahl M, Dormuth CR, Clemens KK, Hu $\mathrm{N}$, Paterson JM, Targownik L, Turin TC, et al. A multicenter observational study of incretin-based drugs and heart failure. N Engl I Med. 2016;374(12):1145-54.

8. Foote C, Perkovic V, Neal B. Effects of SGLT2 inhibitors on cardiovascular outcomes. Diabetes Vasc Dis Res. 2012;9(2):117-23.

9. Green JB, Bethel MA, Armstrong PW, Buse JB, Engel SS, Garg J, Josse R, Kaufman KD, Koglin J, Korn S, et al. Effect of sitagliptin on cardiovascular outcomes in type 2 diabetes. N Engl J Med. 2015;373(3):232-42.

10. Scirica BM, Bhatt DL, Braunwald E, Steg PG, Davidson J, Hirshberg B, Ohman P, Frederich R, Wiviott SD, Hoffman EB, et al. Saxagliptin and cardiovascular outcomes in patients with type 2 diabetes mellitus. N Engl Med. 2013:369(14):1317-26.

11. Toh S, Hampp C, Reichman ME, Graham DJ, Balakrishnan S, Pucino F, Hamilton J, Lendle S, lyer A, Rucker M, et al. Risk for hospitalized heart failure among new users of saxagliptin, sitagliptin, and other antihyperglycemic drugs: a retrospective cohort study. Ann Intern Med. 2016;164(11):705-14.

12. Zannad F, Cannon CP, Cushman WC, Bakris GL, Menon V, Perez AT, Fleck PR, Mehta CR, Kupfer S, Wilson C, et al. Heart failure and mortality outcomes in patients with type 2 diabetes taking alogliptin versus placebo in EXAMINE: a multicentre, randomised, double-blind trial. Lancet. 2015;385(9982):2067-76.

13. Zinman B, Wanner C, Lachin JM, Fitchett D, Bluhmki E, Hantel S, Mattheus M, Devins T, Johansen OE, Woerle HJ, et al. Empagliflozin, cardiovascular outcomes, and mortality in type 2 diabetes. N Engl J Med. 2015:373(22):2117-28. 
14. Pharmacologic approaches to glycemic treatment. Diabetes Care. 2017:40(Suppl 1):S64-74.

15. FDA approves Jardiance to reduce cardiovascular death in adults with type 2 diabetes. http://www.fda.gov/NewsEvents/Newsroom/PressAnnouncements/ucm531517.htm. Accessed 15 Dec 2016.

16. Fadini GP, Avogaro A, Degli Esposti L, Russo P, Saragoni S, Buda S, Rosano G, Pecorelli S, Pani L. Risk of hospitalization for heart failure in patients with type 2 diabetes newly treated with DPP-4 inhibitors or other oral glucose-lowering medications: a retrospective registry study on 127,555 patients from the Nationwide OsMed Health-DB database. Eur Heart J. 2015;36(36):2454-62.

17. Kim SC, Glynn RJ, Liu J, Everett BM, Goldfine AB. Dipeptidyl peptidase-4 inhibitors do not increase the risk of cardiovascular events in type 2 diabetes: a cohort study. Acta Diabetol. 2014;51(6):1015-23.

18. Velez M, Peterson EL, Wells K, Swadia T, Sabbah HN, Williams LK, Lanfear $D E$. Association of antidiabetic medications targeting the glucagon-like peptide 1 pathway and heart failure events in patients with diabetes. J Card Fail. 2015;21(1):2-8.

19. FDA drug safety communication: FDA adds warnings about heart failure risk to labels of type 2 diabetes medicines containing saxagliptin and alogliptin. http://www.fda.gov/Drugs/DrugSafety/ucm486096.htm. Accessed 7 Sept 2016

20. Ray WA. Evaluating medication effects outside of clinical trials: new-user designs. Am J Epidemiol. 2003;158(9):915-20.

21. Deyo RA, Cherkin DC, Ciol MA. Adapting a clinical comorbidity index for use with ICD-9-CM administrative databases. J Clin Epidemiol. 1992;45(6):613-9.

22. Young BA, Lin E, Von Korff M, Simon G, Ciechanowski P, Ludman EJ, Everson-Stewart S, Kinder L, Oliver M, Boyko EJ, et al. Diabetes complications severity index and risk of mortality, hospitalization, and healthcare utilization. Am J Manag Care. 2008;14(1):15-23.

23. Fairman $\mathrm{K}, \mathrm{Motheral} B$. Evaluating medication adherence: which measure is right for your program? J Manag Care Pharm. 2000;6(6):499-504.

24. Austin PC. Using the standardized difference to compare the prevalence of a binary variable between two groups in observational research. Commun Stat Simul Comput. 2009;38(6):1228-34.

25. Austin PC, Stuart EA. Moving towards best practice when using inverse probability of treatment weighting (IPTW) using the propensity score to estimate causal effects in observational studies. Stat Med. 2015;34(28):3661-79.

26. Parsons LS. Reducing bias in a propensity score matched-pair sample using greedy matching techniques. In: Proceedings of the twenty-sixth annual SAS users group international conference: 2001. Long Beach: SAS Institute, Inc.; 2001. p. 214-26.

27. Austin PC. The performance of different propensity score methods for estimating marginal hazard ratios. Stat Med. 2013;32:2837-49.

28. Fewell Z, Hernan MA, Wolfe F, Tilling K, Choi H, Sterne JAC. Controlling for time-dependent confounding using marginal structural models. Stata J. 2004;4(4):402-20.

29. Robins JM, Hernan MA, Brumback B. Marginal structural models and causal inference in epidemiology. Epidemiology. 2000;11(5):550-60.

30. Eurich DT, Weir DL, Simpson SH, Senthilselvan A, McAlister FA. Risk of new onset heart failure in patients using sitagliptin: a population-based cohort study. Diabet Med. 2016;33(5):621-30.

31. Fu AZ, Johnston SS, Ghannam A, Tsai K, Cappell K, Fowler R, Riehle E, Cole AL, Kalsekar I, Sheehan J. Association between hospitalization for heart failure and dipeptidyl peptidase 4 inhibitors in patients with type 2 diabetes: an observational study. Diabetes Care. 2016;39(5):726-34.

32. Yu OH, Filion KB, Azoulay L, Majdan A, Suissa S. Incretin-based drugs and the risk of congestive heart failure. Diabetes Care. 2015;38(2):277-84.

33. Monami M, Dicembrini I, Mannucci E. Dipeptidyl peptidase-4 inhibitors and heart failure: a meta-analysis of randomized clinical trials. Nutr Metab Cardiovasc Dis. 2014;24(7):689-97.
34. Savarese G, Perrone-Filardi P, D'Amore C, Vitale C, Trimarco B, Pani L, Rosano GM. Cardiovascular effects of dipeptidyl peptidase-4 inhibitors in diabetic patients: a meta-analysis. Int J Cardiol. 2015;181:239-44.

35. Wu S, Hopper I, Skiba M, Krum H. Dipeptidyl peptidase-4 inhibitors and cardiovascular outcomes: meta-analysis of randomized clinical trials with 55,141 participants. Cardiovasc Ther. 2014;32(4):147-58.

36. Neal B, Perkovic V, Mahaffey KW, De Zeeuw D, Fulcher G, Erondu N, Shaw W, Law G, Desai M, Matthews D. Canagliflozin and cardiovascular and renal events in type 2 diabetes. N Engl J Med. 2017. doi:10.1056/NEJMoa1611925 (published online ahead of print June 12, 2017).

37. Kosiborod M, Cavender MA, Fu AZ, Wilding JP, Khunti K, Holl RW, Norhammar A, Birkeland KI, Jørgensen M, Thuresson M, et al. Lower risk of heart failure and death in patients initiated on SGLT-2 inhibitors versus other glucose-lowering drugs: the CVD-REAL study. Circulation. 2017. doi:10.1161/CIRCULATIONAHA.117.029190 (published online ahead of print May 18, 2017)

38. Ampudia-Blasco FJ, Romera I, Arino B, Gomis R. Following the results of the EMPA-REG OUTCOME trial with empagliflozin, is it possible to speak of a class effect? Int J Gen Med. 2017;10:23-6.

39. Sattar N, McLaren J, Kristensen SL, Preiss D, McMurray JJ. SGLT2 inhibition and cardiovascular events: why did EMPA-REG outcomes surprise and what were the likely mechanisms? Diabetologia. 2016;59(7):1333-9.

40. Multicenter trial to evaluate the effect of dapagliflozin on the incidence of cardiovascular events (DECLARE-TIMI58). https://clinicaltrials.gov/ct2/ show/NCT01730534. Accessed 23 Mar 2017.

41. Sonesson C, Johansson PA, Gause-Nilsson I. Cardiovascular effects of dapagliflozin in patients with type 2 diabetes and different risk categories: a meta-analysis. Cardiovasc Diabetol. 2016;15:37.

42. Nakamura T, Iwanaga Y, Miyaji Y, Nohara R, Ishimura T, Miyazaki S. Cardiovascular efficacy of sitagliptin in patients with diabetes at high risk of cardiovascular disease: a 12-month follow-up. Cardiovasc Diabetol. 2016;15:54.

43. Abdul-Ghani M, Del Prato S, Chilton R, DeFronzo RA. SGLT2 inhibitors and cardiovascular risk: lessons learned from the EMPA-REG OUTCOME study. Diabetes Care. 2016;39(5):717-25.

44. Martens P, Mathieu C, Verbrugge FH. Promise of SGLT2 inhibitors in heart failure: diabetes and beyond. Curr Treat Options Cardiovasc Med. 2017:19(3):23.

45. Kusaka H, Koibuchi N, Hasegawa Y, Ogawa H, Kim-Mitsuyama S. Empagliflozin lessened cardiac injury and reduced visceral adipocyte hypertrophy in prediabetic rats with metabolic syndrome. Cardiovasc Diabetol. 2016:15:157.

46. Saine ME, Carbonari DM, Newcomb CW, Nezamzadeh MS, Haynes K, Roy JA, Cardillo S, Hennessy S, Holick CN, Esposito DB, et al. Determinants of saxagliptin use among patients with type 2 diabetes mellitus treated with oral anti-diabetic drugs. BMC Pharmacol Toxicol. 2015;16:8.

47. ClinicalTrials.gov. https://clinicaltrials.gov/ct2/results?term =SGLT2+heart +failure\&Search=Search. Accessed 23 Mar 2017.

48. Standards of medical care in diabetes-2017: summary of revisions. Diabetes Care. 2017:40(Supppl 1):S4-S5.

49. Handelsman Y, Bloomgarden ZT, Grunberger G, Umpierrez G, Zimmerman RS, Bailey TS, Blonde L, Bray GA, Cohen AJ, Dagogo-Jack S, et al. American Association of Clinical Endocrinologists and American College of Endocrinology — clinical practice guidelines for developing a diabetes mellitus comprehensive care plan-2015. Endocr Pract. 2015;21(Suppl 1):1-87.

50. Heman MA, Brumbeack B, Robins JM. Marginal structural models to estimate the causal effect of zidovudine on the survival of HIV-positive men. Epidemiology. 2000;11(5):561-70. 